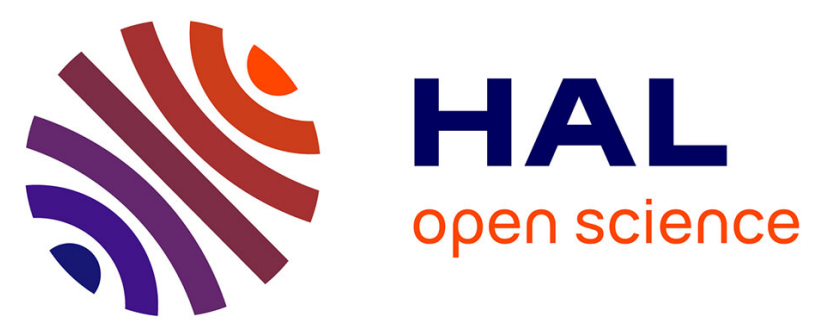

\title{
Persistent Organic Room-Temperature Phosphorescence in Cyclohexane-trans-1,2- Bisphthalimide Derivatives: The Dramatic Impact of Heterochiral vs. Homochiral interactions
}

Ludovic Favereau, Cassandre Quinton, Cyril Poriel, Thierry Roisnel, Denis Jacquemin, Jeanne Crassous

\section{To cite this version:}

Ludovic Favereau, Cassandre Quinton, Cyril Poriel, Thierry Roisnel, Denis Jacquemin, et al.. Persistent Organic Room-Temperature Phosphorescence in Cyclohexane-trans-1,2- Bisphthalimide Derivatives: The Dramatic Impact of Heterochiral vs. Homochiral interactions. Journal of Physical Chemistry Letters, 2020, 11 (15), pp.6426-6434. 10.1021/acs.jpclett.0c01816 . hal-02999452

\section{HAL Id: hal-02999452}

\section{https://hal-univ-rennes1.archives-ouvertes.fr/hal-02999452}

Submitted on 10 Nov 2020

HAL is a multi-disciplinary open access archive for the deposit and dissemination of scientific research documents, whether they are published or not. The documents may come from teaching and research institutions in France or abroad, or from public or private research centers.
L'archive ouverte pluridisciplinaire $\mathbf{H A L}$, est destinée au dépôt et à la diffusion de documents scientifiques de niveau recherche, publiés ou non, émanant des établissements d'enseignement et de recherche français ou étrangers, des laboratoires publics ou privés. 


\title{
Persistent Organic Room-Temperature Phosphorescence in Cyclohexane-trans-1,2- Bisphthalimide Derivatives: The Dramatic Impact of Heterochiral vs. Homochiral interactions
}

\section{Ludovic Favereau, ${ }^{\text {a, }}$, Cassandre Quinton, ${ }^{a}$ Cyril Poriel, a Thierry Roisnel, ${ }^{a}$ Denis Jacquemin, ${ }^{b}$ Jeanne Crassous ${ }^{a}$}

${ }^{a}$ Univ Rennes, CNRS, ISCR-UMR 6226, ScanMAT-UMS 2001, F-35000 Rennes, France. Email: ludovic.favereau@univ-rennes1.fr

${ }^{\mathrm{b}}$ Laboratoire CEISAM, UMR 6230, CNRS, Université de Nantes, Nantes, France.

\begin{abstract}
Persistent metal-free Room-Temperature Phosphorescence (RTP) materials attract significant interest owing to the production of long-lived triplet excited states. Although several organic designs show RTP, the impact of intermolecular interactions on the triplet excitons stabilization and migrations remains hardly understood because obtaining different ordered intermolecular interactions while conserving identical molecular electronic properties is very challenging. We propose here a new strategy to circumvent this problem by taking advantage of the distinct molecular packing that can be found between enantiomer and racemic forms of a chiral molecule. Structural, photophysical and chiroptical investigations of chiral cyclohexane bisphthalimide derivatives showed that heterochiral and homochiral dimer interactions play a crucial role on the triplet excited state stabilization, resulting in higher RTP efficiency for enantiopure systems than for racemic one. This study paves the way to the use of molecular chirality to rationalize supramolecular properties arising from subtle intermolecular interactions.
\end{abstract}

\section{TOC Graphic}

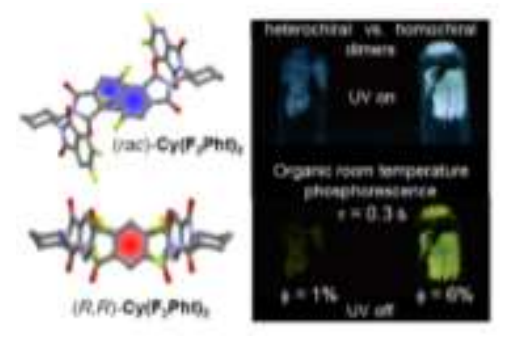

The organization of $\pi$-conjugated molecules in the solid state plays a pivotal role on the photophysical and electronic properties of the obtained material. ${ }^{1,2,3}$ Understanding the relationships between molecular structure, the intermolecular interactions and the resulting 
properties of the supramolecular assembly is therefore of prime importance. ${ }^{4}$ Indeed, the arrangement of molecules within a material does strongly impact many nanoscale phenomena such as light absorption, luminescence, energy transfer, charges conduction or analyte sensing, which ultimately control the overall activity of the resulting macrostructure. In addition, supramolecular assemblies may yield emerging properties owing to specific molecular electronic interactions and organization. In this global framework, persistent metal-free RoomTemperature Phosphorescence (RTP) materials has attracted a growing interest owing to the production of long-lived triplet excited states, relevant for several applications, e.g., optoelectronics (OPVs, OLEDs), bio-imaging, sensing, cryptography, photocatalysis. 5, 6, 7, 8, 9, 10,11 While phosphorescent metal-containing systems are well-known and rely on an efficient inter-system crossing (ISC) process induced by the large spin-obit couplings (SOCs) of metal, ${ }^{12}$, 13 organic heavy-atom-free RTP materials have several advantages, such as lower cost and toxicity, and more tuneable photophysical properties. ${ }^{14,15}$ In organic systems, RTP can typically take place by improving ISC efficiency, which can be done either by increasing the SOCs using heavy halogen atoms such as bromine or iodide, or promoted by the presence of low-lying transitions following El-Sayed's rule. This can be achieved using heterocyles or carbonyl groups for instance. ${ }^{16,17,18}$ Besides these electronic factors, structural rigidity and ordered packing are also crucial parameters to minimize the radiationless deactivation of the ultra-long lived excited triplet states. ${ }^{19,20,21,22,23,24,25,26,27,28}$ An effective understanding of the underlying photophysical process is needed to optimize the design of organic RTP molecular materials, and, more precisely, the impact of intermolecular interactions on the triplet exciton stabilization and migration needs to be understood. Such task is however made difficult, as obtaining different intermolecular interactions while keeping the same molecular electronic properties is often beyond reach, e.g., the addition of methylene substituent in a lateral alkyl chain may induce a completely different solid state packing. ${ }^{25,} 27$ To circumvent this problem, chirality offers a unique possibility to investigate such parameters since enantiomer and racemic forms of a chiral molecule have identical chemical and physical properties in solution, ${ }^{29}$ but may present distinct molecular packing as heterochiral and homochiral dimer interactions might differ, which in turn can yield different photophysical properties and/or electronic conduction. $^{30,31,32,33,34,35,36}$ Accordingly, organic chiral compounds with RTP property modulated by enantiopurity represents an exceptional opportunity for exploring the impact of intermolecular interactions on the efficiency of ultra-long RTP without altering the molecular electronic properties. Moreover, the possibility to obtain RT circularly polarized luminescence (CPL) with chiral organic emitters could bring additional advantages over unpolarized RTP, ${ }^{37}$, 
38, 39 notably in the design of efficient CP-OLEDs, in spintronic, optical information processing, as well as in bioimaging (ellipsometry-based tomography) and chiral sensing, where the state of light polarization (either left or right) may bring higher contrast and selectivity than for an non-polarized light.

During our work on chiral $\pi$-conjugated molecules for chiroptoelectronic applications, ${ }^{30,40,41}$ we serendipitously noticed that enantiopure cyclohexane-trans-1,2-bisphthalimide derivatives, $(R R)-\mathbf{C y}\left(\mathbf{F}_{2} \mathbf{P h t}\right)_{2},{ }^{42}$ display persistent RTP emission process once precipitated in the reaction medium. Irradiation of the obtained solid by UV light at $\lambda=365 \mathrm{~nm}$ (benchtop UV lamp) affords light blue luminescence, which evolves in a yellow one, seen by the naked eye upon switching off the excitation light (Scheme 1). This behaviour prompted us to explore the possible impact of molecular enantiopurity on the RTP efficiency. Herein we report our results regarding the study of chiral organic persistent RTP molecular materials based on cyclohexane tetrafluorinated derivatives $\left((R R)-,(S S)_{-}\right.$and $(\mathrm{rac})-\mathbf{C y}\left(\mathbf{F}_{2} \mathbf{P h t}\right)_{2}$, Figure 1). Crystals of $\mathbf{C y}\left(\mathbf{F}_{2} \mathbf{P h t}\right)_{2}$ display ultra-long yellow phosphorescence, which is dependent on both the enantiopurity of the molecular components and the temperature. While enantiopure and racemic crystals exhibit persistent phosphorescence at $77 \mathrm{~K}$, only the former shows efficient RTP under ambient (air) conditions with a lifetime of $0.3 \mathrm{~s}$. X-ray analysis, chiroptical, and photophysical characterizations reveal key differences in the stabilization of the triplet states within the different crystal arrangements of the enantiopure and racemic compounds. This study represents an unprecedented example of chiral persistent RTP materials in which enantiopurity controls the emission efficiency by modifying the crystal intermolecular packing of sterically and electronically identical individual components.

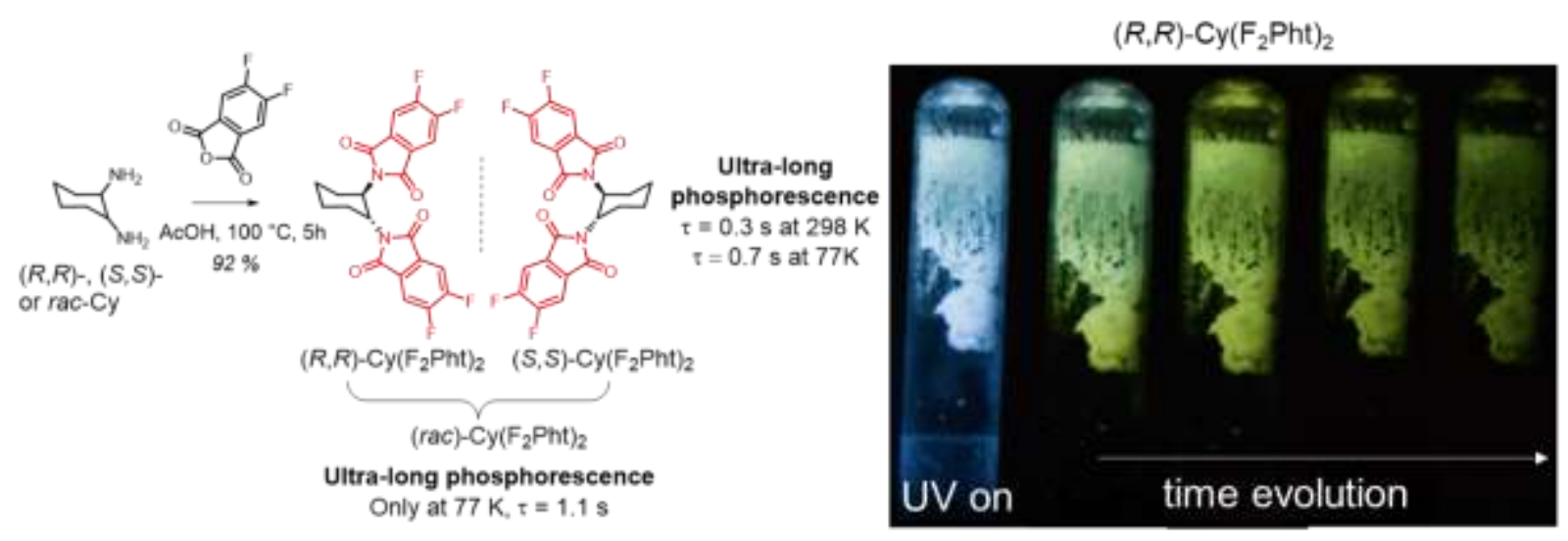

Scheme 1. Synthesis of (RR)-, $(S S)$ - and (rac $)-\mathbf{C y}\left(\mathbf{F}_{2} \mathbf{P h t}\right)_{2}$ with their main photophysical characteristics and picture of the RTP process for $(R R)-\mathbf{C y}\left(\mathbf{F}_{2} \mathbf{P h t}\right)_{2}$, under UV light excitation at $\lambda=365 \mathrm{~nm}$ (benchtop UV lamp) and after switched it off (time evolution). 
$(R R)_{-},(S S)$ - and (rac)-Cy( $\left.\mathbf{F} \mathbf{2} \mathbf{P h t}\right)_{2}$ were efficiently synthetized by mixing one equivalent of corresponding $(R R)$-, $(S S)$ - or ( $r a c)$-cyclohexane-trans-1,2-diamine with excess of 5,6difluoroisobenzofuran-1,3-dione in acetic acid in 92\% yield for the three compounds (Scheme 1). These chiral derivatives were fully characterized by NMR, chiral HPLC and mass spectrometry, ${ }^{42}$ displaying characteristic signals of both phthalimide and cyclohexane units in the aromatic and aliphatic parts of the ${ }^{1} \mathrm{H}$ spectrum, respectively, with a $C_{2}$ symmetry for the bisphthalimide moieties (see the SI for details). ${ }^{43,44}$ Crystals of both $(R R)$ - and $(r a c)-\mathbf{C y}\left(\mathbf{F}_{2} \mathbf{P h t}\right)_{2}$ have been obtained by slow diffusion of pentane vapours into chloroform solutions and their resulting X-ray structures show distinct features regarding the molecular geometry and the supramolecular arrangement: $(R R)$ - and $(\mathrm{rac})-\mathbf{C y}\left(\mathbf{F}_{2} \mathbf{P h t}\right)_{2}$ crystallize in the $C 222_{1}$ and $P \overline{1}$ space group, respectively (Figure 1 and table S1), with the expected equatorial conformation for the phthalimide substituents attached to the cyclohexane backbone. While the two phthalimide planes form an angle of $c a .56^{\circ}$ in the racemic compound, the enantiopure $(R R)-\mathbf{C y}\left(\mathbf{F}_{2} \mathbf{P h t}\right)_{2}$ shows a more open structure with an angle of $71^{\circ}$ (Figures 1a and 1b). In addition, different N1-C1-C2-N2 torsion angles are found in (rac)- and $(R R)-\mathbf{C y}\left(\mathbf{F}_{2} \mathbf{P h t}\right)_{2}: 49.3^{\circ}$ and $60.6^{\circ}$, respectively, resulting in a less face-to-face phthalimides arrangement for the former. These significant differences may be attributed to symmetry reasons, which sometimes induce different packing between pure enantiomers and corresponding racemic mixture. ${ }^{29}$ While ( $\mathrm{rac}$ )and $(R R)-\mathbf{C y}\left(\mathbf{F}_{2} \mathbf{P h t}\right)_{2}$ form similar phthalimide dimers in a head-to-tail fashion, separated by 3.46 and $3.53 \AA$, respectively (Figures $1 \mathrm{c}$ and 1d), expanding the packing to the supramolecular level reveals important differences between both crystals. Indeed, $(R R)-\mathbf{C y}\left(\mathbf{F}_{2} \mathbf{P h t}\right)_{2}$ dimers exhibit a continuous intermolecular chain along the $\mathrm{x}$ axis, maintained by identical $\pi-\pi$ interactions involving each phthalimide unit (Figure 1f), whereas ( $r a c)-\mathbf{C y}\left(\mathbf{F}_{2} \mathbf{P h t}\right)_{2}$ dimers present $\pi-\pi$ intermolecular interactions along the $\mathrm{y}$ axis with other dimer neighbours (blue colour, Figure 1e). In this additional dimer interaction, the phthalimide groups are separated by $3.45 \AA$ and appear much less co-facially oriented than the previously described red one, affording distinct molecular arrangements for homo- and heterochiral dimer interactions. Further single-crystal X-ray difraction analysis show that for the red dimers, the angle between transition dipoles and the interconnected axis is larger than the critical value of $54.7^{\circ}(\theta=71.5$ and $77.6^{\circ}$ for $(\mathrm{rac})$ - and $(R R)-\mathbf{C y}\left(\mathbf{F}_{2} \mathbf{P h t}\right)_{2}$, respectively, Figure 1c,d), indicating the presence of $\mathrm{H}$-aggregates (see the SI for details), while for the blue dimer, this angle is around $55.4^{\circ}$ at the boarder between $\mathrm{H}$ - and J-aggregates states. ${ }^{16,45,46}$ Fluorine atoms might have an indirect effect on the overserved solid-state arrnagement since in the crystal structure, one fluorine atom 
shows a short contact bond with an aromatic proton of a phthalimide fragment neighbors for $(R R)_{-} \mathbf{C y}\left(\mathbf{F}_{2} \mathbf{P h t}\right)_{2}$ or with an alkyl proton of the cyclohexane core in the case of $\operatorname{rac}-\mathbf{C y}\left(\mathbf{F}_{2} \mathbf{P h t}\right)_{2}$ (Figure 1), suggesting an effect on the molecular packing .

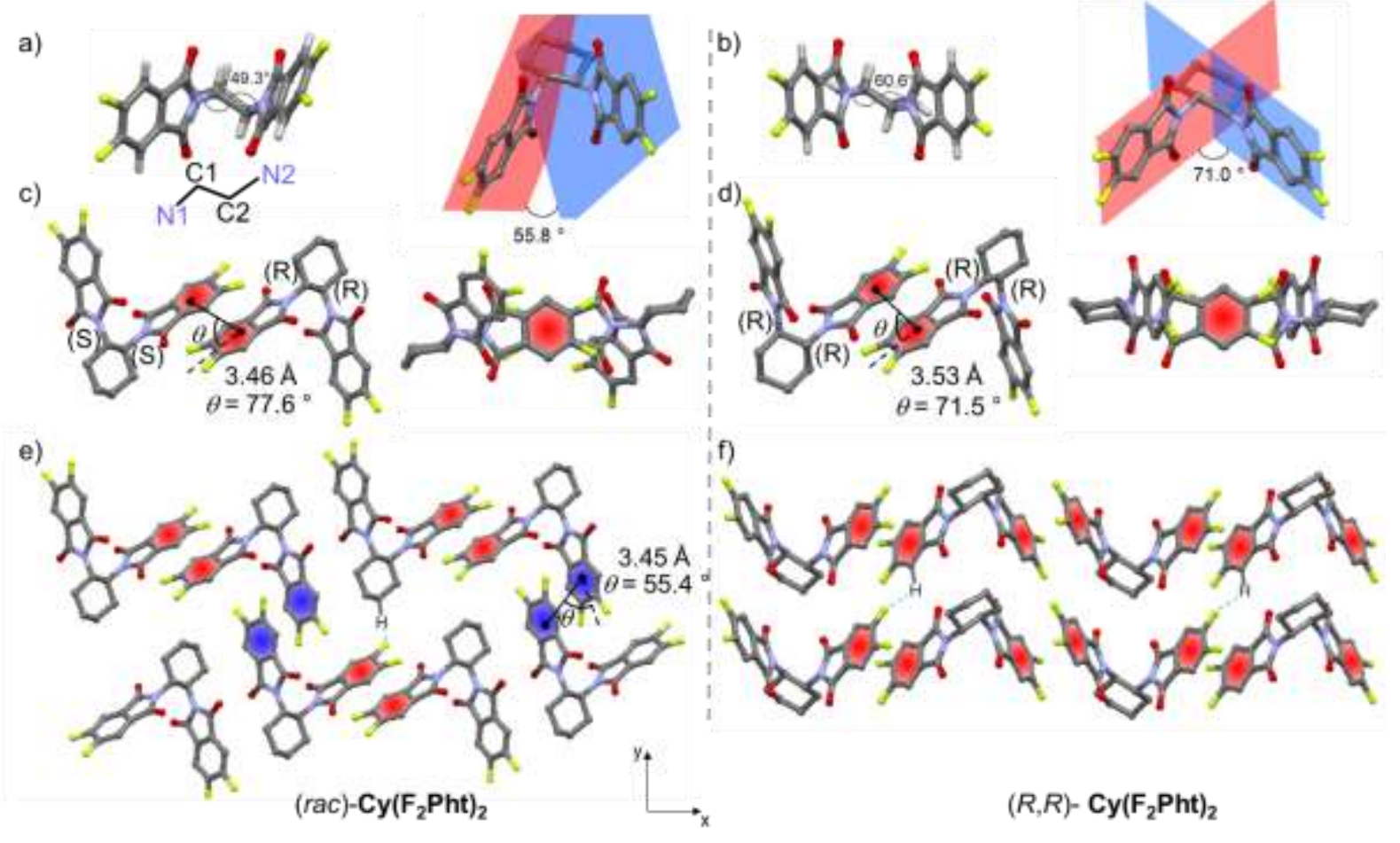

Figure 1. Comparison of the X-ray crystallographic structures of ( $\mathrm{rac})-\mathbf{C y}\left(\mathbf{F}_{2} \mathbf{P h t}\right)_{2}$ (left, a) and $(R R)-\mathbf{C y}\left(\mathbf{F}_{2} \mathbf{P h t}\right)_{2}$ (right, b) at the molecular level with N1-C1-C2-N2 dihedral angle and angle between phthalimide plans; and at the supramolecular level with different views of intermolecular interactions between dimers, c) and d); and supramolecular packing e) and f). $\theta$ angles represent the angle between the transition dipoles and interconnected axis of ( $\mathrm{rac}$ )$\mathbf{C y}\left(\mathbf{F}_{2} \mathbf{P h t}\right)_{2}$ and $(R R)-\mathbf{C y}\left(\mathbf{F}_{2} \mathbf{P h t}\right)_{2}$ with values of $71.5^{\circ}$ and $77.6^{\circ}$, respectively for the red dimers, evidencing the presence of $\mathrm{H}$ aggregates, in comparison to the blue dimer $\left(\theta=55.4^{\circ}\right.$, see SI for additional details).

The UV-vis spectrum of $\mathbf{C y}\left(\mathbf{F}_{2} \mathbf{P h t}\right)_{2}$ in dilute solution $\left(10^{-5} \mathrm{M}\right)$ displays an intense absorption bands at ca. $230 \mathrm{~nm}\left(\varepsilon=5.5 \times 10^{4} \mathrm{M}^{-1} \mathrm{~cm}^{-1}\right)$ and smaller broader one at $285 \mathrm{~nm}\left(\varepsilon=2.0 \times 10^{3}\right.$ $\mathrm{M}^{-1} \mathrm{~cm}^{-1}$ ), respectively assigned to $\pi-\pi^{*}$ and $\mathrm{n}-\pi^{*}$ transitions (Figure 2). ${ }^{43}$ At $298 \mathrm{~K}$ in dichloromethane solution, $\mathbf{C y}\left(\mathbf{F}_{2} \mathbf{P h t}\right)_{2}$ exhibits a very weak luminescence at $c a .310 \mathrm{~nm}(\phi<$ 0.01 ), presumably due to an efficient ISC process from the singlet to the triplet excited state of the molecule, as already reported for phthalimide derivatives. ${ }^{47}$ Indeed, lowering the temperature to $77 \mathrm{~K}$ affords a residual fluorescence signal at $300 \mathrm{~nm}$ and mainly phosphorescence emission, with a maximum at $461 \mathrm{~nm}$ and a lifetime of $c a .1 \mathrm{~s}$ arising from $\pi$ - 
$\pi^{*}$ triplet state, in line with the phosphorescence emission reported for phthalimide derivatives (Figures 2 and S9). To gain further details, theoretical calculations were performed on $\mathbf{C y}\left(\mathbf{F}_{2} \mathbf{P h t}\right)_{2}$ (see the SI). Upon light excitation, population of excited $\mathbf{C y}\left(\mathbf{F}_{2} \mathbf{P h t}\right)_{2}$ singlet states $\left(S_{\mathrm{n}}\right)$ occurs, followed by internal conversion and relaxation to the lowest excited singlet state $\left(S_{1}\right.$, Figure 2). From the latter, a residual fluorescence emission occurs experimentally recorded at $312 \mathrm{~nm}$, which is well reproduced by the calculations (319 nm, Figure 2). This radiative deactivation process is in competition with the ISC process leading to $\mathbf{C y}\left(\mathbf{F}_{2} \mathbf{P h t}\right)_{2}$ triplet excited states. Theoretical calculations indicate the presence of eight triplet states close to $S_{1}$ in energy, with moderate to rather strong spin orbit coupling with values up to 4.3 and $4.6 \mathrm{~cm}^{-1}$ for the $\mathrm{S} 1 \rightarrow \mathrm{T} 6$ and $\mathrm{S} 1 \rightarrow \mathrm{T} 5$ transitions. These SOC are of significant intensity for organic systems without heavy atom and highlight the potential of this molecular system to investigate phosphorescence emission, which was also well reproduced by theoretical calculation at 447 nm (Figure 2). For comparison purpose, mono phatalimide derivative $\mathbf{C y F}_{2} \mathbf{P h t}$ was also synthetized and its optical properties characterized. $\mathbf{C y F}_{2} \mathbf{P h t}$ displays almost the same UV-vis absorption and luminescence profiles than those of $\mathbf{C y}\left(\mathbf{F}_{2} \mathbf{P h t}\right)_{2}$, notably a similar low temperature phosphorescence emission at $460 \mathrm{~nm}$ with a lifetime of $c a .0 .8 \mathrm{~s}$. These results suggest a weak electronic interaction between the two phthalimide units in $\mathbf{C y}\left(\mathbf{F}_{\mathbf{2}} \mathbf{P h t}\right)_{2}$, consistent with their arrangements (see Figure 1) and with its molar extinction coefficients twice as high as for $\mathbf{C y F}_{\mathbf{2}} \mathbf{P h t}$. As expected, the cyclohexane-trans-1,2-bisamido linker has a limited role of in term of photophysics due to its $s p^{3}$ core but remains crucial as it brings the property of chirality owing to the presence of the two enantiomers ( $R R$ and $S S$ ) for this conformation. 

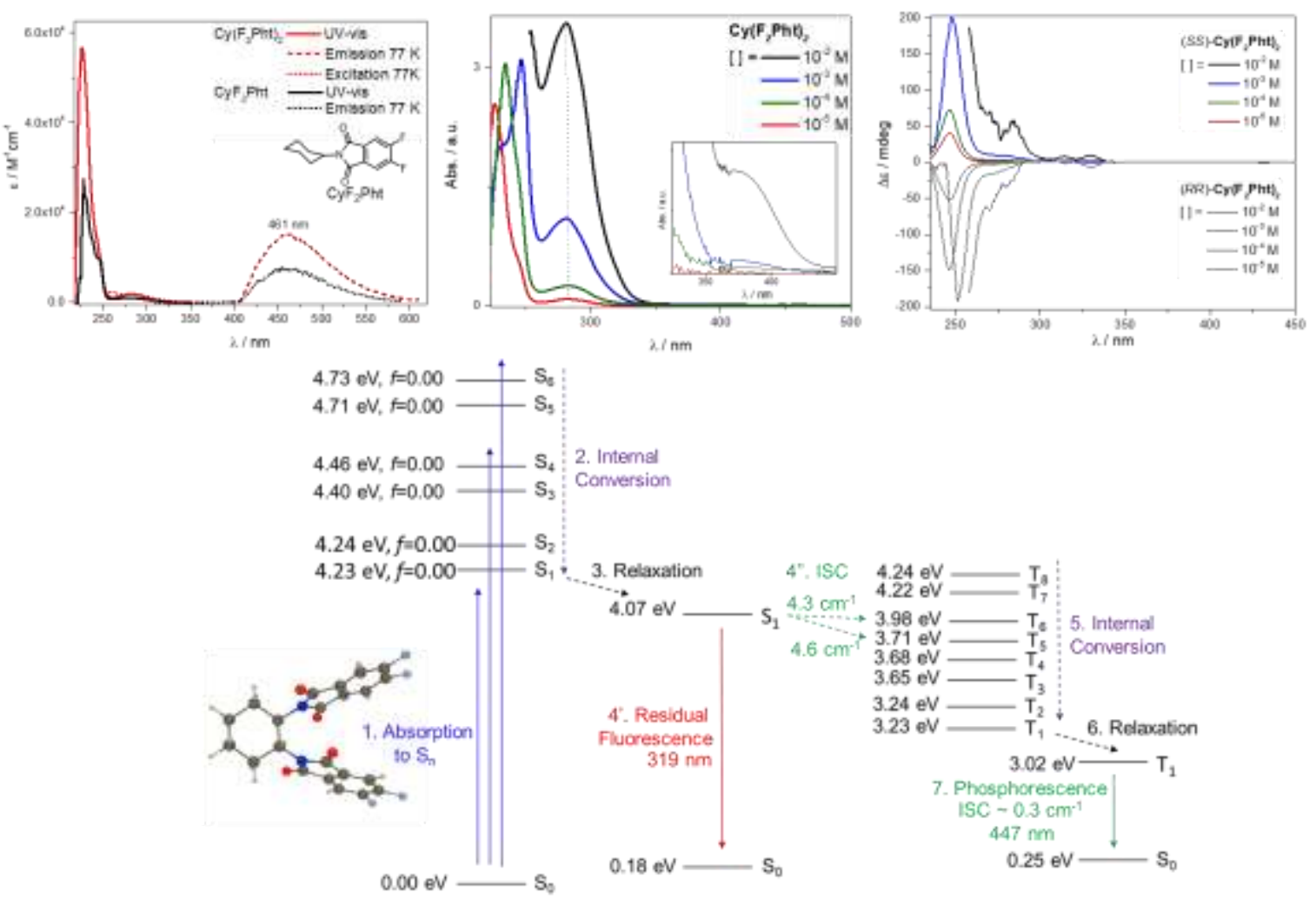

Figure 2. Top: left: UV-vis absorption spectrum of $\mathbf{C y}\left(\mathbf{F}_{2} \mathbf{P h t}\right)_{2}$ and $\mathbf{C y F}_{2} \mathbf{P h t}$ in dichloromethane solution $\left(\sim 10^{-5} \mathrm{M}\right.$, red and black, respectively) at $298 \mathrm{~K}$ together with their emission spectrum recorded in 2-MeTHF at $77 \mathrm{~K}$ (red and black dashed lines, respectively, $\lambda_{\text {exci }}$ $=280 \mathrm{~nm})$ and the corresponding excitation spectrum of $\mathbf{C y}\left(\mathbf{F}_{2} \mathbf{P h t}\right)_{2}$ (red dotted line, $\lambda_{\text {exci }}=$ $460 \mathrm{~nm})$; middle: UV-vis absorption spectra of $\mathbf{C y}\left(\mathbf{F}_{2} \mathbf{P h t}\right)_{2}$ recorded at different concentration: $10^{-5}$ (red), $10^{-4}$ (green), $10^{-3}$ (blue) and $10^{-2} \mathrm{M}$ (black) in dichloromethane solution at $298 \mathrm{~K}$; right: Electronic Circular Dichroism spectra of $(R R)$ - and $(S S)-\mathbf{C y}_{\mathbf{y}}\left(\mathbf{F}_{2} \mathbf{P h t}\right)_{2}$ recorded at different concentration: $10^{-5}$ (red), $10^{-4}$ (green), $10^{-3}$ (blue) and $10^{-2} \mathrm{M}$ (black) in dichloromethane solution at $298 \mathrm{~K}$ (due to rapid signal saturation upon increasing concentration, only the $230-450 \mathrm{~nm}$ region is displayed). Bottom: Energy-level diagrams and calculated ISC channels from the ground state $S_{0}$ to the singlet excited states $\left(S_{n}\right)$ and finally the triplet excited states $\left(T_{n}\right)$ with the most significant corresponding spin-orbit coupling (SOC) constants.

Further insights have been obtained by recording the Electronic Circular Dichroism (ECD) of $(R R)$ - and $(S S)-\mathbf{C y}\left(\mathbf{F}_{2} \mathbf{P h t}\right)_{2}$ (Figure S7), which exhibited the expected mirror-image spectra with positive intense bands at 230 and $242 \mathrm{~nm}\left(\Delta \varepsilon \sim 35 \mathrm{M}^{-1} \mathrm{~cm}^{-1}\right)$, arising from two chiral excitonic couplings between the perpendicular in-plane electronic dipoles present in each phthalimide unit, ${ }^{43}$ and an additional very weak transition around $280 \mathrm{~nm}$ for $(S S)-\mathbf{C y}\left(\mathbf{F}_{2} \mathbf{P h t}\right)_{2}$ 
$\left(\Delta \varepsilon \sim-0.5 \mathrm{M}^{-1} \mathrm{~cm}^{-1}\right)$. Increasing solution concentrations up to $10^{-2} \mathrm{M}$ does not induce drastic changes in the UV-vis signature and mainly resulted in a small $15 \mathrm{~nm}$ red-shift of the band at $230 \mathrm{~nm}$, a proportional intensity increase of the $n-\pi^{*}$ transitions absorption at ca. $285 \mathrm{~nm}$ with a slight blue-shift of $3 \mathrm{~nm}$, and the apparition of a weak band between 340 and $400 \mathrm{~nm}$ when the concentration attains $10^{-3}-10^{-2} \mathrm{M}$ (Figure 2), suggesting the presence of intermolecular interactions. Likewise, ECD of concentrated solutions reveals the appearance of very weak mirror-image signals up to $350 \mathrm{~nm}$, marked by two positive maxima at 315 and $330 \mathrm{~nm}$ for $(S S)-\mathbf{C y}\left(\mathbf{F}_{2} \mathbf{P h t}\right)_{2}$ (Figure 2), while the rest of the ECD spectrum becomes above the detection range of the detector. The obtained X-ray crystallographic structures and the slight blue-shift of the absorption spectra upon increasing concentration are consistent with a predominant contribution of $\mathrm{H}$-aggregates between intermolecular phthalimide fragments, which have been identified as an important parameter for stabilization of triplet excitons and RTP in organic systems. ${ }^{14,15,17,21}$ Similar experiments on monophtalimide model compound, $\mathbf{C y F}_{2} \mathbf{P h t}$, gives identical optical signature for concentrated solution (Figures S13-S15), which confirms the involvement of intermolecular interactions. Such aggregation behaviour also modifies the luminescence properties of $\mathbf{C y}\left(\mathbf{F}_{2} \mathbf{P h t}\right)_{2}$ since the emission spectra display significant changes at high concentration including a decrease of the low emissive band around $310 \mathrm{~nm}$ (Figure 3a), concomitantly with the appearance of weakly luminescence around $420 \mathrm{~nm}$, presumably arising from H-type dimers. Indeed, excitation of the sample at $360 \mathrm{~nm}$, i.e., in the absorption band attributed to intermolecular interactions, results in similar fluorescence emission at $425 \mathrm{~nm}(\tau$ $=2.2$ and $12 \mathrm{~ns}$, Figure 3b). Moreover, excitation spectra at $425 \mathrm{~nm}$ clearly indicate that this emission band involved molecular and intermolecular optical transitions at 260-280 nm and between 340 and $400 \mathrm{~nm}$, respectively (Figure S8). Overall, these photophysical characterizations in solution show the tendency of $\mathbf{C y}\left(\mathbf{F}_{2} \mathbf{P h t}\right)_{2}$ to form weakly fluorescent aggregates at high concentration, as expected for H-type molecular interactions, ${ }^{48}$ but do not afford any evidence of phosphorescence emission, nor of different signatures of the racemic and enantiopure supramolecular assemblies. 
a)

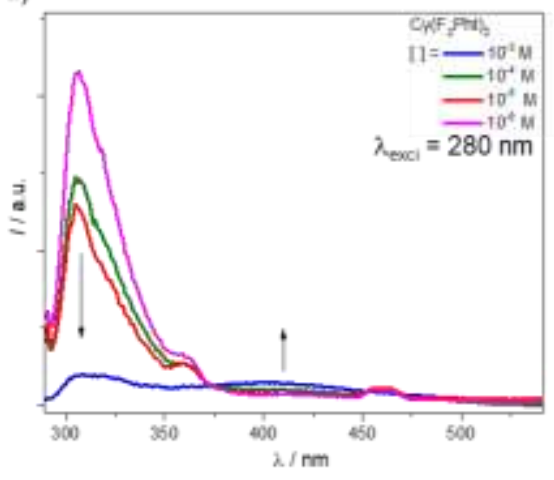

b)

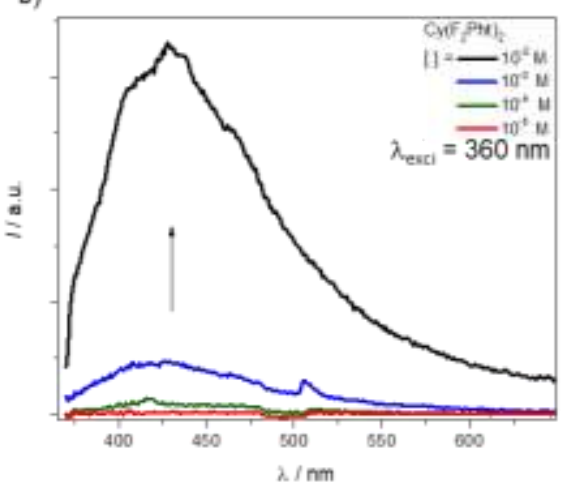

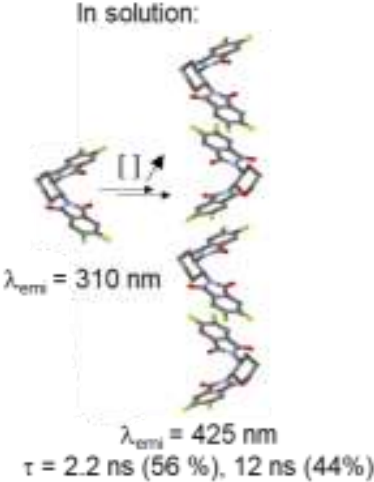

Figure 3. a) Luminescence spectra of $\mathbf{C y}\left(\mathbf{F}_{2} \mathbf{P h t}\right)_{2}$ recorded at different concentrations: $10^{-6}$ (purple), $10^{-5}$ (red), $10^{-4}$ (green) and $10^{-3} \mathrm{M}$ (blue) in dichloromethane solution at $298 \mathrm{~K}\left(\lambda_{\text {exci }}=\right.$ $280 \mathrm{~nm})$; b) luminescence spectra of $\mathbf{C y}\left(\mathbf{F}_{2} \mathbf{P h t}\right)_{2}$ recorded at different concentration: $10^{-5}$ (red), $10^{-4}$ (green), $10^{-3}$ (blue) and $10^{-2} \mathrm{M}$ (black) in dichloromethane solution at $298 \mathrm{~K}\left(\lambda_{\text {exci }}=360\right.$ $\mathrm{nm}$ ) with illustration of the presumably molecular aggregation in highly concentrated solutions.

The luminescence properties of the $(R R)_{-},(S S)-,($ rac $)-\mathbf{C y}\left(\mathbf{F}_{2} \mathbf{P h t}\right)_{2}$ crystals are displayed in Figure 4. At $298 \mathrm{~K}$ in air atmosphere, $(\mathrm{rac})-\mathbf{C y}\left(\mathbf{F}_{2} \mathbf{P h t}\right)_{2}$ crystals excited at $330 \mathrm{~nm}$ give a weak and broad emission spectrum with two hardly distinguishable and similarly intense bands at 435 and $530 \mathrm{~nm}$. When excitation takes place at $350 \mathrm{~nm}$, a similar luminescence profile is observed but with a slightly higher emission intensity for the $435 \mathrm{~nm}$ emission band. Such change becomes clearer when the crystals are excited at $370 \mathrm{~nm}$, corresponding to the intermolecular interactions from the phthalimide aggregates, since the signal at $530 \mathrm{~nm}$ appears only as a shoulder of the main emission process at $435 \mathrm{~nm}$. In comparison, while excitation of $(R R)-\mathbf{C y}\left(\mathbf{F}_{2} \mathbf{P h t}\right)_{2}$ crystals at $370 \mathrm{~nm}$ affords a similar emission spectrum with a maximum at $440 \mathrm{~nm}$, the excitations at 330 and $350 \mathrm{~nm}$ result in different luminescence profiles. Indeed, upon excitation at $350 \mathrm{~nm},(R R)-\mathbf{C y}\left(\mathbf{F}_{2} \mathbf{P h t}\right)_{2}$ displays two well resolved emission bands at 440 and $525 \mathrm{~nm}$, the latter being of higher intensity, which strikingly differ from $(\mathrm{rac})-\mathbf{C y}\left(\mathbf{F}_{2} \mathbf{P h t}\right)_{2}$. Finally, the predominance of the emission process at $525 \mathrm{~nm}$ is even more pronounced when the enantiopure crystals are excited at $330 \mathrm{~nm}\left(\mathrm{I}^{530} / \mathrm{I}^{425}\right.$ ratio of 14$)$. Importantly, yellow RTP remains clearly present after switching off the excitation source at all these excitation wavelengths, suggesting that this process may be related to the emission band at $530 \mathrm{~nm}$. Indeed, emission spectra of $(R R)-\mathbf{C y}\left(\mathbf{F}_{2} \mathbf{P h t}\right)_{2}$ crystals excited at $350 \mathrm{~nm}$ and recorded after 10, 30 and $50 \mathrm{~ms}$ of delay afford an identical vibronic responses with maxima at 496 and $532 \mathrm{~nm}$. To gain further insights, time-resolved luminescence measurements have been recorded at these two luminescence maxima. At $530 \mathrm{~nm}$, a very long lifetime $(\tau)$ of $\sim 0.3 \mathrm{~s}$ was detected as the 
main decay component for the RTP process whereas at $435 \mathrm{~nm}$, only a short nanosecond lifetime was recorded (Figure S9). Such measurements allowed us to assign the two observed emission bands at 435 and $530 \mathrm{~nm}$ to singlet $\left(\mathrm{S}_{1}\right)$ and triplet $\left(\mathrm{T}_{1}\right)$ emissions of the $(R R)$ $\mathbf{C y}\left(\mathbf{F}_{2} \mathbf{P h t}\right)_{2}$ aggregates, since the former is close to the fluorescence observed in concentrated solution (Figure 3) whereas the phosphorescence at $530 \mathrm{~nm}$ appears redshifted as compared to the molecular phosphorescence recorded in frozen solution of diluted $\mathbf{C y}\left(\mathbf{F}_{2} \mathbf{P h t}\right)_{2}$ at $77 \mathrm{~K}$ (Figure 2).
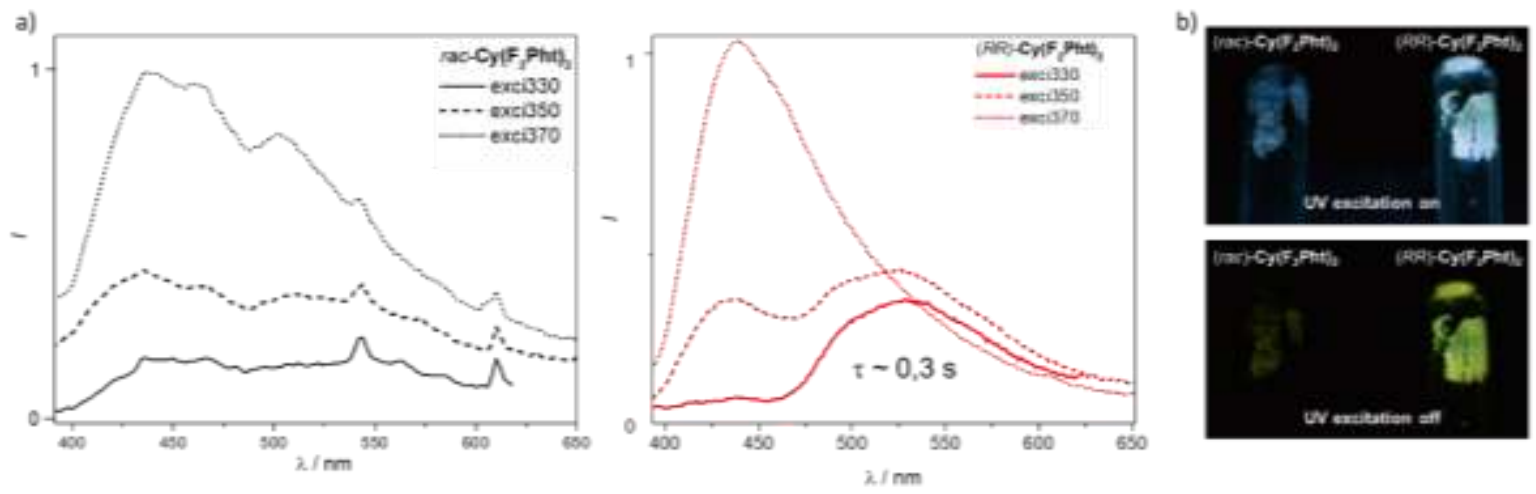

c)

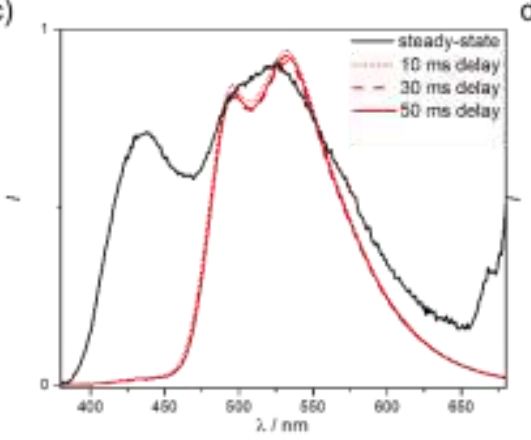

d)

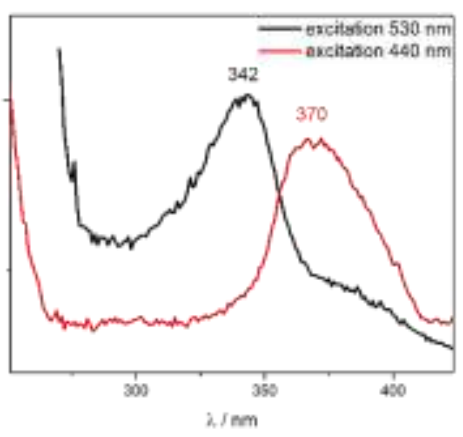

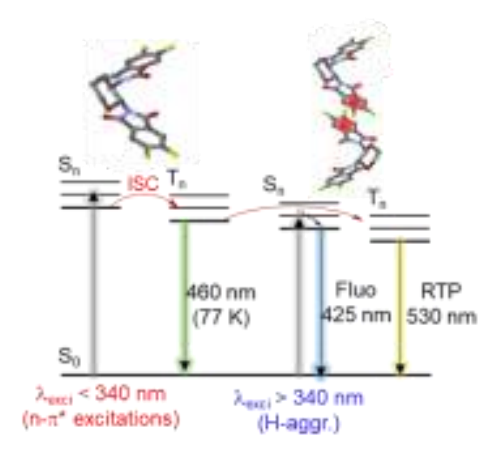

Figure 4. a) Luminescence spectra of ( $\mathrm{rac})-\mathbf{C y}\left(\mathbf{F}_{2} \mathbf{P h t}\right)_{2}$ crystals (left, black lines) and (RR)$\mathbf{C y}\left(\mathbf{F}_{2} \mathbf{P h t}\right)_{2}$ (right, red lines) recorded at different wavelength excitations: 330 (solid line), 350 (dashed line) and $370 \mathrm{~nm}$ (dotted line) at $298 \mathrm{~K}$; b) top: pictures showing luminescence of ( $\mathrm{rac}$ )$\mathbf{C y}\left(\mathbf{F}_{2} \mathbf{P h t}\right)_{2}$ (left) and $(R R)-\mathbf{C y}\left(\mathbf{F}_{2} \mathbf{P h t}\right)_{2}$ (right) under UV light excitation with benchtop UV lamp and bottom: just after switched it off; c) steady-state (black solid line) and delayed emission spectra (red lines, $\lambda_{\text {exci }}=280 \mathrm{~nm}$ ), recorded at different times delays: $50 \mathrm{~ms}$ (solid line), $30 \mathrm{~ms}$ (dashed line) and $10 \mathrm{~ms}$ (dotted line) at $298 \mathrm{~K}$; d) left: excitation of luminescence of $(R R)-\mathbf{C y}\left(\mathbf{F}_{2} \mathbf{P h t}\right)$ recorded at 440 (red) and $525 \mathrm{~nm}$ (black) and right: proposed mechanism of RTP generation with impact of excitation wavelengths.

We have also investigated the luminescence excitation spectra at 440 and $525 \mathrm{~nm}$. The latter notably indicated that the electronic transitions responsible for the fluorescence and 
phosphorescence emissions involve different absorption bands, which may be assigned to intermolecular aggregates $(350-410 \mathrm{~nm})$ and molecular absorption bands $(\lambda<350 \mathrm{~nm})$, respectively (Figure $4 \mathrm{c}$ ), and suggest that the observed RTP process is more efficiently triggered when light excitations occurs on electronic transitions of the molecular unit (for $\lambda_{\text {exc }}$ $<350 \mathrm{~nm}$ ). According to photophysical studies of phthalimides, ${ }^{43,} 47$ the lower part of their absorption spectrum $(280-340 \mathrm{~nm})$ is dominated by $\mathrm{n}-\pi^{*}$ electronic transitions, which are associated to a high ISC rate between the singlet ${ }^{1}\left(n-\pi^{*}\right)$ and lowest triplet ${ }^{3}\left(\pi, \pi^{*}\right)$ excited states in accordance with El-Sayed's rule, ${ }^{49}$ and as evidenced here by the recorded phosphorescence process in diluted phthalimide solution at $77 \mathrm{~K}$. Such fast ISC process should occur in both racemic and enantiopure crystals of $\mathbf{C y}\left(\mathbf{F}_{2} \mathbf{P h t}\right)_{2}$ when exciting the molecular electronic transitions $\left(\lambda_{\text {exc }}<350 \mathrm{~nm}\right)$. However, when one excites the absorption band related to the intermolecular aggregates, that is, for $\lambda_{\mathrm{exc}}>350 \mathrm{~nm}$, the ISC rate drops and fluorescence becomes the main radiative deactivation process. Interestingly, the racemic and enantiopure crystals did not emit with the same intensity, as can be seen in Figure 4. To ascertain such difference, a similar photophysical study was performed on $(S S)-\mathbf{C y}\left(\mathbf{F}_{2} \mathbf{P h t}\right)_{2}$ and afforded, as expected, similar results as $(R R)-\mathbf{C y}\left(\mathbf{F}_{2} \mathbf{P h t}\right)_{2}$ (see the SI for detail). Further, quantitative evidences of the impact of enantiopurity on the RTP efficiency were provided by luminescence quantum yield measurements recorded at different wavelengths. Both enantiopure crystals show close emission efficiencies with significant values of 2-3 and 3-6\% when exciting either at 350 or $370 \mathrm{~nm}$, respectively, while low quantum yields of 0.5 and $1 \%$ were measured for (rac)-Cy(F2 $\mathbf{F h t})_{2}$ at 350 or $370 \mathrm{~nm}$ respectively, unambiguously reflecting the more efficient RTP process in enantiopure crystal. This fact is also confirmed by naked eyes since the yellow RTP is less bright and shorter than the one observed from enantiopure crystals (see videos in the SI).
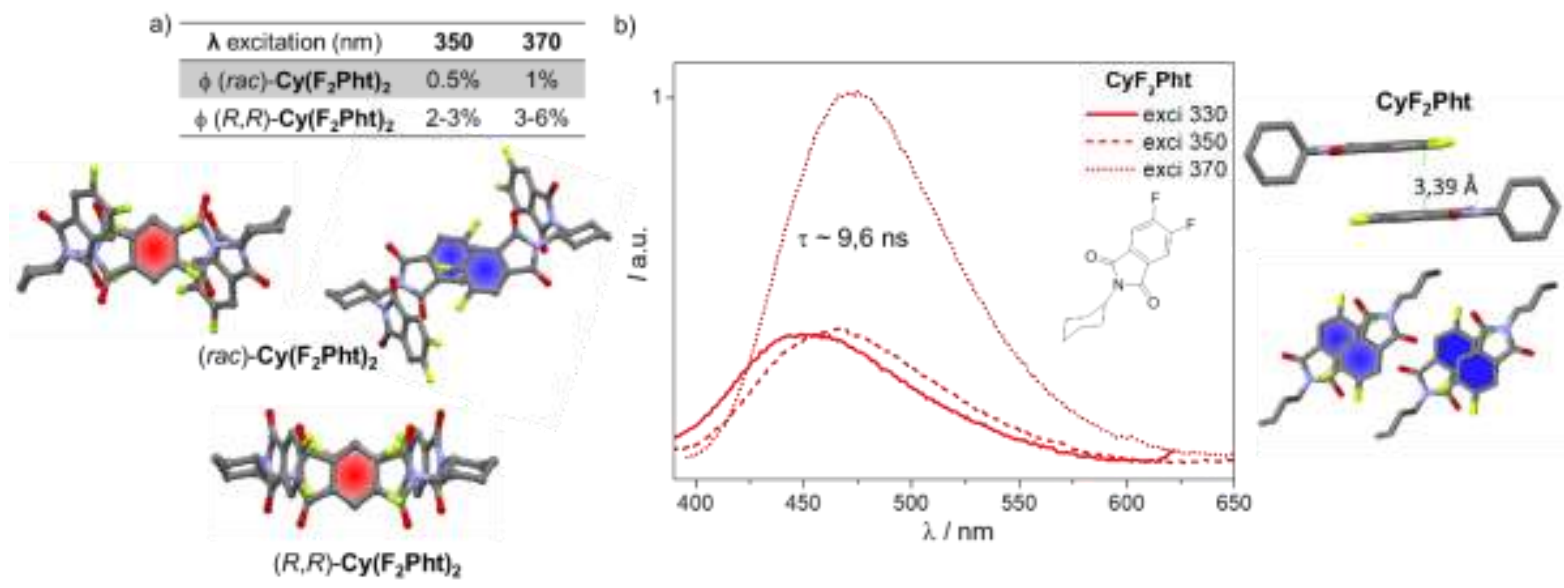

$R, R)-\mathrm{Cy}\left(\mathrm{F}_{2} \mathrm{Pht}\right)_{2}$ 
Figure 5. a) Luminescence quantum yields of $(R R)-\mathbf{C y}\left(\mathbf{F}_{2} \mathbf{P h t}\right)_{2}$ and (rac $)-\mathbf{C y}\left(\mathbf{F}_{2} \mathbf{P h t}\right)_{2}$ crystals recorded at 350 and $370 \mathrm{~nm}$ at $298 \mathrm{~K}$; b) Luminescence spectra of $\mathbf{C y F}_{2} \mathbf{P h t}$ crystals recorded at different wavelength excitations: 310 (solid line), 350 (dashed line) and $370 \mathrm{~nm}$ (dotted line) at $298 \mathrm{~K}$ with X-ray crystallographic structures of intermolecular dimers.

Since the photophysical experiments were performed on crystals, the RTP process can be directly related to the presence of the illustrated intermolecular red dimers of phthalimide fragments in Figure 1. As mentioned above, these dimers show characteristics of H-type aggregates, which have been identified as a stabilization strategy for triplet excitons and an efficient approach to get RTP in organic systems. ${ }^{16,20,21}$ Indeed, photophysical experiments performed on crystals of model compound $\mathbf{C y F}_{2} \mathbf{P h t}$ did not reveal any RTP process, which could be explained by the lack of "red" H-aggregates found in $\mathbf{C y}\left(\mathbf{F}_{2} \mathbf{P h t}\right)_{2}$ (Figure 5). Accordingly, the higher RTP efficiency in enantiopure $\mathbf{C y}\left(\mathbf{F}_{2} \mathbf{P h t}\right)_{2}$ can be explained by the greater presence of such interactions in comparison with ( $\mathrm{rac})-\mathbf{C y}\left(\mathbf{F}_{2} \mathbf{P h t}\right)_{2}$, highlighting the subtle and crucial role of the heterochiral and homochiral dimer interactions, ultimately leading to distinct molecular packing, and thus different RTP efficiency. Indeed, the presence of additional "blue" dimers for $(\mathrm{rac})-\mathbf{C y}\left(\mathbf{F}_{2} \mathbf{P h t}\right)_{2}$ result in less H-type aggregates and a less ordered molecular network, which presumably decreases the stabilization of triplet excitons, as for $\mathbf{C y F}_{2} \mathbf{P h t}$. Such parameter was tentatively investigated by carrying out some photophysical experiments at $77 \mathrm{~K}$ to probe the impact of temperature on the stabilization of the phosphorescence arising from the supramolecular assembly (Figure S11-S12). Reducing the temperature clearly stabilizes and enhances the long emission process over the fluorescence one for both ( $r a c)-\mathbf{C y}\left(\mathbf{F}_{2} \mathbf{P h t}\right)_{2}$ and $(R R)-\mathbf{C y}\left(\mathbf{F}_{2} \mathbf{P h t}\right)_{2}$, which exhibited phosphorescence at $490 \mathrm{~nm}$ with a lifetime of 1.1 and 0.7 s., respectively. When light excitation is performed at $350 \mathrm{~nm}$, only weak fluorescence is observed for $(\mathrm{rac})-\mathbf{C y}\left(\mathbf{F}_{2} \mathbf{P h t}\right)_{2}$ at $400 \mathrm{~nm}$, indicating a critical role of the temperature on the deactivation of the triplet excited state for such derivative. Unfortunately, no circularly polarized RTP was observed from enantiopure crystals despite several attempts, presumably due to the low value of polarized emission in these derivatives.

We have reported RTP process in chiral metal free tetrafluorinated cyclohexane-trans-1,2naphthalimide derivatives whose efficiency is modulated by the enantiopurity of the molecular component. This surprising aspect was assigned to a different organization of homo- and heterochiral dimers in the solid state, which has a crucial impact on the presence of H-type aggregates within the crystal network, as unambiguously ascertained by X-ray analysis. Such 
symmetry parameter results in an efficient RTP for enantiopure crystal of $\mathbf{C y}\left(\mathbf{F}_{2} \mathbf{P h t}\right)_{2}$ with a quantum yield of $3 \%$ and a lifetime of $0.3 \mathrm{~s}$ while, in contrast, ( rac $)-\mathbf{C y}\left(\mathbf{F}_{2} \mathbf{P h t}\right)_{2}$ displays a quantum yield of $0.5 \%$ only under ambient (air) conditions. Photophysical and chiroptical measurements afforded further insights on the structural parameters that stabilized triplet excitons within these chiral molecular networks and notably the crucial impact of intermolecular $\mathrm{H}$-aggregates and temperature. These findings clearly highlight an interesting feature of chiral molecular materials on the modulation of supramolecular material properties by the degree of enantiopurity. It is our hope that these results may help in designing more efficient chiral RTP materials in order to obtain circularly polarized phosphorescence in metal free compounds.

\section{Acknowledgments}

We acknowledge the Ministère de l'Education Nationale, de la Recherche et de la Technologie, the Centre National de la Recherche Scientifique (CNRS). The PRISM core facility (Biogenouest $@$, UMS Biosit, Université de Rennes 1 - Campus de Villejean - 35043 RENNES Cedex, FRANCE) is acknowledged for the ECD measurements. The authors thanked Dr. N. Vanthuyne for the chiral HPLC analysis. Guillaume Juin is warmly thanked for his movie on the room temperature process.

\section{Supporting Information}

Additional detailed experimental information, synthesis, and graphs can be find in the Electronic Supporting Information.

\section{References}

1. Riera-Galindo, S.; Tamayo, A.; Mas-Torrent, M., Role of Polymorphism and Thin-Film Morphology in Organic Semiconductors Processed by Solution Shearing. ACS Omega 2018, 3 (2), 2329-2339.

2. Ostroverkhova, O., Organic Optoelectronic Materials: Mechanisms and Applications. Chem. Rev. 2016.

3. Beaujuge, P. M.; Fréchet, J. M. J., Molecular Design and Ordering Effects in $\pi$ Functional Materials for Transistor and Solar Cell Applications. J. Am. Chem. Soc. 2011, 133 (50), 20009-20029.

4. Aida, T.; Meijer, E. W., Supramolecular Polymers - we've Come Full Circle. Isr. J. Chem. 2020, 60 (1-2), 33-47.

5. Hirata, S.; Totani, K.; Zhang, J.; Yamashita, T.; Kaji, H.; Marder, S. R.; Watanabe, T.; Adachi, C., Efficient Persistent Room Temperature Phosphorescence in Organic Amorphous Materials under Ambient Conditions. Adv. Funct. Mater. 2013, 23 (27), 3386-3397. 
6. Mukherjee, S.; Thilagar, P., Recent advances in purely organic phosphorescent materials. Chem. Commun. 2015, 51 (55), 10988-11003.

7. Ma, H.; Lv, A.; Fu, L.; Wang, S.; An, Z.; Shi, H.; Huang, W., Room-Temperature Phosphorescence in Metal-Free Organic Materials. Annalen der Physik 2019, 531 (7), 1800482. 8. Chen, H.; Yao, X.; Ma, X.; Tian, H., Amorphous, Efficient, Room-Temperature Phosphorescent Metal-Free Polymers and Their Applications as Encryption Ink. Adv. Opt. Mater. 2016, 4 (9), 1397-1401.

9. Wang, J.; Liang, J.; Xu, Y.; Liang, B.; Wei, J.; Li, C.; Mu, X.; Ye, K.; Wang, Y., Purely Organic Phosphorescence Emitter-Based Efficient Electroluminescence Devices. J. Phys. Chem. Lett. 2019, 10 (19), 5983-5988.

10. Zhang, T.; Ma, X.; Wu, H.; Zhu, L.; Zhao, Y.; Tian, H., Molecular Engineering for Metal-Free Amorphous Materials with Room-Temperature Phosphorescence. Angew. Chem. Int. Ed. Engl. 2020, 59 (28), 11206-11216.

11. Kabe, R.; Adachi, C., Organic long persistent luminescence. Nature 2017, 550 (7676), 384-387.

12. Xu, H.; Chen, R.; Sun, Q.; Lai, W.; Su, Q.; Huang, W.; Liu, X., Recent progress in metal-organic complexes for optoelectronic applications. Chem. Soc. Rev. 2014, 43 (10), 32593302.

13. Henwood, A. F.; Zysman-Colman, E., Lessons learned in tuning the optoelectronic properties of phosphorescent iridium(iii) complexes. Chem. Commun. 2016.

14. Data, P.; Takeda, Y., Recent Advancements in and the Future of Organic Emitters: TADF- and RTP-Active Multifunctional Organic Materials. Chem. Asian J. 2019, 14 (10), 1613-1636.

15. Kenry; Chen, C.; Liu, B., Enhancing the performance of pure organic room-temperature phosphorescent luminophores. Nat. Commun. 2019, 10 (1), 2111.

16. An, Z.; Zheng, C.; Tao, Y.; Chen, R.; Shi, H.; Chen, T.; Wang, Z.; Li, H.; Deng, R.; Liu, X.; Huang, W., Stabilizing triplet excited states for ultralong organic phosphorescence. Nat Mater 2015, 14 (7), 685-690.

17. Hirata, S., Recent Advances in Materials with Room-Temperature Phosphorescence: Photophysics for Triplet Exciton Stabilization. Adv. Opt. Mater. 2017, 5 (17), 1700116-n/a.

18. Ma, H.; Peng, Q.; An, Z.; Huang, W.; Shuai, Z., Efficient and Long-Lived RoomTemperature Organic Phosphorescence: Theoretical Descriptors for Molecular Designs. J. Am. Chem. Soc. 2019, 141 (2), 1010-1015.

19. Chen, X.; Xu, C.; Wang, T.; Zhou, C.; Du, J.; Wang, Z.; Xu, H.; Xie, T.; Bi, G.; Jiang, J.; Zhang, X.; Demas, J. N.; Trindle, C. O.; Luo, Y.; Zhang, G., Versatile Room-TemperaturePhosphorescent Materials Prepared from N-Substituted Naphthalimides: Emission Enhancement and Chemical Conjugation. Angew. Chem. Int. Ed. 2016, 55 (34), 9872-9876.

20. Lucenti, E.; Forni, A.; Botta, C.; Carlucci, L.; Giannini, C.; Marinotto, D.; Pavanello, A.; Previtali, A.; Righetto, S.; Cariati, E., Cyclic Triimidazole Derivatives: Intriguing Examples of Multiple Emissions and Ultralong Phosphorescence at Room Temperature. Angew Chem Int Ed 2017, 56 (51), 16302-16307.

21. Lucenti, E.; Forni, A.; Botta, C.; Carlucci, L.; Giannini, C.; Marinotto, D.; Previtali, A.; Righetto, S.; Cariati, E., H-Aggregates Granting Crystallization-Induced Emissive Behavior and Ultralong Phosphorescence from a Pure Organic Molecule. J. Phys. Chem. Lett. 2017, 8 (8), 1894-1898.

22. Xie, Y.; Ge, Y.; Peng, Q.; Li, C.; Li, Q.; Li, Z., How the Molecular Packing Affects the Room Temperature Phosphorescence in Pure Organic Compounds: Ingenious Molecular Design, Detailed Crystal Analysis, and Rational Theoretical Calculations. Adv. Mater. 2017, 29 (17), 1606829-n/a. 
23. Bian, L.; Shi, H.; Wang, X.; Ling, K.; Ma, H.; Li, M.; Cheng, Z.; Ma, C.; Cai, S.; Wu, Q.; Gan, N.; Xu, X.; An, Z.; Huang, W., Simultaneously Enhancing Efficiency and Lifetime of Ultralong Organic Phosphorescence Materials by Molecular Self-Assembly. J. Am. Chem. Soc. 2018, 140 (34), 10734-10739.

24. Gu, L.; Shi, H.; Miao, C.; Wu, Q.; Cheng, Z.; Cai, S.; Gu, M.; Ma, C.; Yao, W.; Gao, Y.; An, Z.; Huang, W., Prolonging the lifetime of ultralong organic phosphorescence through dihydrogen bonding. J. Mater. Chem. C 2018.

25. Huang, R.; Ward, J. S.; Kukhta, N. A.; Avó, J.; Gibson, J.; Penfold, T.; Lima, J. C.; Batsanov, A. S.; Berberan-Santos, M. N.; Bryce, M. R.; Dias, F. B., The influence of molecular conformation on the photophysics of organic room temperature phosphorescent luminophores. J. Mater. Chem. C 2018, 6 (34), 9238-9247.

26. Mu, Y.; Yang, Z.; Chen, J.; Yang, Z.; Li, W.; Tan, X.; Mao, Z.; Yu, T.; Zhao, J.; Zheng, S.; Liu, S.; Zhang, Y.; Chi, Z.; Xu, J.; Aldred, M. P., Mechano-induced persistent roomtemperature phosphorescence from purely organic molecules. Chem. Sci. 2018.

27. Zhang, Z.; Tang, L.; Fan, X.; Wang, Y.; Zhang, K.; Sun, Q.; Zhang, H.; Xue, S.; Yang, W., N-Alkylcarbazoles: homolog manipulating long-lived room-temperature phosphorescence. J. Mater. Chem. C 2018, 6 (33), 8984-8989.

28. Wang, J.; Chai, Z.; Wang, J.; Wang, C.; Han, M.; Liao, Q.; Huang, A.; Lin, P.; Li, C.; Li, Q.; Li, Z., Mechanoluminescence or Room-Temperature Phosphorescence: Molecular Packing-Dependent Emission Response. Angew. Chem. Int. Ed. 2019, 58 (48), 17297-17302.

29. J. Jacques, A. C., S. H. Wilen, Enantiomers, Racemates, \& Resolutions. J. Wiley \& Sons, New York: 1981.

30. Josse, P.; Favereau, L.; Shen, C.; Dabos-Seignon, S.; Blanchard, P.; Cabanetos, C.; Crassous, J., Enantiopure versus Racemic Naphthalimide End-Capped Helicenic Non-fullerene Electron Acceptors: Impact on Organic Photovoltaics Performance. Chem. Eur. J. 2017, 23 (26), 6277-6281.

31. Hatakeyama, T.; Hashimoto, S.; Oba, T.; Nakamura, M., Azaboradibenzo[6]helicene: Carrier Inversion Induced by Helical Homochirality. J. Am. Chem. Soc. 2012, 134 (48), 1960019603.

32. Réthoré, C.; Avarvari, N.; Canadell, E.; Auban-Senzier, P.; Fourmigué, M., Chiral Molecular Metals: Syntheses, Structures, and Properties of the AsF6- Salts of Racemic ( \pm -, (R)-, and (S)-Tetrathiafulvalene-Oxazoline Derivatives. J. Am. Chem. Soc. 2005, 127 (16), 5748-5749.

33. Pop, F.; Auban-Senzier, P.; Frąckowiak, A.; Ptaszyński, K.; Olejniczak, I.; Wallis, J. D.; Canadell, E.; Avarvari, N., Chirality Driven Metallic versus Semiconducting Behavior in a Complete Series of Radical Cation Salts Based on Dimethyl-Ethylenedithio-Tetrathiafulvalene (DM-EDT-TTF). J. Am. Chem. Soc. 2013, 135 (45), 17176-17186.

34. Brandt, J. R.; Salerno, F.; Fuchter, M. J., The added value of small-molecule chirality in technological applications. Nat. Rev. Chem. 2017, 1 (6), 0045.

35. Yang, Y.; Rice, B.; Shi, X.; Brandt, J. R.; Correa da Costa, R.; Hedley, G. J.; Smilgies, D. M.; Frost, J. M.; Samuel, I. D. W.; Otero-de-la-Roza, A.; Johnson, E. R.; Jelfs, K. E.; Nelson, J.; Campbell, A. J.; Fuchter, M. J., Emergent Properties of an Organic Semiconductor Driven by its Molecular Chirality. ACS Nano 2017, 11 (8), 8329-8338.

36. Rice, B.; LeBlanc, L. M.; Otero-de-la-Roza, A.; Fuchter, M. J.; Johnson, E. R.; Nelson, J.; Jelfs, K. E., A computational exploration of the crystal energy and charge-carrier mobility landscapes of the chiral [6] helicene molecule. Nanoscale 2018, 10 (4), 1865-1876.

37. Chen, W.; Tian, Z.; Li, Y.; Jiang, Y.; Liu, M.; Duan, P., Long-Persistent Circularly Polarized Phosphorescence from Chiral Organic Ionic Crystals. Chem. Eur. J. 2018, 24 (66), 17444-17448. 
38. Liang, X.; Liu, T. T.; Yan, Z. P.; Zhou, Y.; Su, J.; Luo, X. F.; Wu, Z. G.; Wang, Y.; Zheng, Y. X.; Zuo, J. L., Organic Room-Temperature Phosphorescence with Strong Circularly Polarized Luminescence Based on Paracyclophanes. Angew. Chem. Int. Ed. 2019, 58 (48), 17220-17225.

39. Hirata, S.; Vacha, M., Circularly Polarized Persistent Room-Temperature Phosphorescence from Metal-Free Chiral Aromatics in Air. J. Phys. Chem. Lett. 2016, 7 (8), 1539-1545.

40. Bouvier, R.; Durand, R.; Favereau, L.; Srebro-Hooper, M.; Dorcet, V.; Roisnel, T.; Vanthuyne, N.; Vesga, Y.; Donnelly, J.; Hernandez, F.; Autschbach, J.; Trolez, Y.; Crassous, J., Helicenes Grafted with 1,1,4,4-Tetracyanobutadiene Moieties: pi-Helical Push-Pull Systems with Strong Electronic Circular Dichroism and Two-Photon Absorption. Chem. Eur. J. 2018, 24 (54), 14484-14494.

41. Dhbaibi, K.; Favereau, L.; Srebro-Hooper, M.; Jean, M.; Vanthuyne, N.; Zinna, F.; Jamoussi, B.; Di Bari, L.; Autschbach, J.; Crassous, J., Exciton coupling in diketopyrrolopyrrole-helicene derivatives leads to red and near-infrared circularly polarized luminescence. Chem. Sci. 2018, 9 (3), 735-742.

42. Li, M.; Li, S. H.; Zhang, D.; Cai, M.; Duan, L.; Fung, M. K.; Chen, C. F., Stable Enantiomers Displaying Thermally Activated Delayed Fluorescence: Efficient OLEDs with Circularly Polarized Electroluminescence. Angew Chem Int Ed 2018, 57 (11), 2889-2893.

43. Gawronski, J.; Kazmierczak, F.; Gawronska, K.; Rychlewska, U.; Nordén, B.; Holmén, A., Excited States of the Phthalimide Chromophore and Their Exciton Couplings: A Tool for Stereochemical Assignments. J. Am. Chem. Soc. 1998, 120 (46), 12083-12091.

44. Gawroński, J.; Brzostowska, M.; Kacprzak, K.; Kołbon, H.; Skowronek, P., Chirality of aromatic bis-imides from their circular dichroism spectra. Chirality 2000, 12 (4), 263-268.

45. Kasha, M.; Rawls, H. R.; El-Bayoumi, M. A., The exciton model in molecular spectroscopy. Pure Appl. Chem. 1965, 11 (3-4), 371-392.

46. Eisfeld, A.; Briggs, J. S., The J- and H-bands of organic dye aggregates. Chemical Physics 2006, 324 (2), 376-384.

47. Wintgens, V.; Valat, P.; Kossanyi, J.; Biczok, L.; Demeter, A.; Bérces, T., Spectroscopic properties of aromatic dicarboximides. Part1.- $\mathrm{N}-\mathrm{H}$ and N-methyl-substituted naphthalimides. J. Chem. Soc., Faraday Trans. 1994, 90 (3), 411-421.

48. Lakowicz, J. R., Principles of Fluorescence Spectroscopy. Springer, New York: 2006.

49. Lower, S. K.; El-Sayed, M. A., The Triplet State and Molecular Electronic Processes in Organic Molecules. Chem. Rev. 1966, 66 (2), 199-241. 